\title{
ON CERTAIN CHARACTERISTICS OF $k$-DIMENSIONAL VARIETIES IN $r$-SPACE
}

BY B. C. WONG

An algebraic variety of $k$ dimensions in $r$-space has numerous characteristics besides its order $n$. The characteristics of algebraic curves and surfaces and the relations they satisfy are known. In this paper we consider a variety $V_{k}$ of dimension $k$ greater than 2. Assuming it to be the complete intersection of $r-k$ hypersurfaces of orders $n_{1}, n_{2}, \cdots, n_{r-k}$ respectively in $S_{r}$, we derive the formulas for a few of its characteristics in terms of the $n$ 's and incidentally obtain the relations connecting them. To avoid unnecessary length of discussion we consider somewhat in detail the $V_{3}$ in $S_{7}$ only and then give the results without demonstration for $V_{k}$ in $S_{r}$ * The method here employed is the familiar one of complete degeneration which we have repeatedly made use of elsewhere in dealing with problems of similar nature. $\dagger$

Now for the purpose of enumerating the characteristics of $V_{k}$ and obtaining their relations we may regard the variety as belonging to an $S_{2 k+1}$, for a $V_{k}$ belonging to an $S_{r}$ where $r>2 k+1$ possesses no characteristics not possessed by a $V_{k}$ of $S_{2 k+1}$. If we project $V_{k}$ from a general $S_{t-1}$ of $S_{2 k+1}$ on to an $S_{2 k+1-t}$

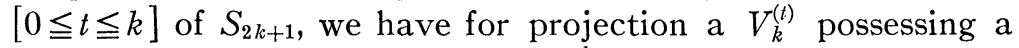
double $(t-1)$-dimensional variety $V_{t-1}^{b_{t-1}}$ of order $b_{t-1}$ and a pinch $(t-2)$-dimensional variety $V_{i-2}^{j t-2}$ of order $j_{t-2}$ lying on $V_{t-1}^{b_{t-1}}$. From a general point of $S_{2 k+1-t}$ we can construct $\infty^{t}$ lines forming a $(t+1)$-dimensional cone of order $b_{t}$ each meeting $V_{k}{ }^{(t)}$ in two distinct points. We say that $V_{k}^{(l)}$ has an apparent double $V_{t}^{b_{t}}$ of order $b_{t}$. Again, from a general point of $S_{2 k+1-t}$ a $t$-dimensional cone of $\infty^{t-1}$ lines of order $j_{t-1}$ can be constructed tangent to

* Some work has been done along this line. See C. Segre, Mehrdimensionale Räume, Encyklopädie der Mathematischen Wissenschaften, $\mathrm{III}_{2}, 7$, pp. 922927.

$\dagger \mathrm{B}$. C. Wong, On the number of apparent multiple points of varieties in hyperspace, this Bulletin, vol. 36, pp. 102-106; and On surfaces in spaces of four and five dimensions, this Bulletin, vol. 36, pp. 861-866. 
$V_{k}^{(t)}$. The projection $V_{k}^{(t+1)}$ of $V_{k}^{(t)}$ in an $S_{2 k-t}$ has a double variety $V_{t}^{b}$ which is the projection of the apparent double variety $V_{t}^{b_{t}}$ of $V_{k}^{(t)}$ and a pinch variety $V_{t-1}^{j_{t-1}}$ which is the section of the tangent $t$-dimensional cone of $V_{k}^{(t)}$ by $S_{2 k-t}$.

If we allow $t$ to take on all the values from 0 to $k$, we obtain $2 k$ characteristics $b_{0}, b_{1}, \cdots, b_{k-1} ; j_{0}, j_{1}, \cdots, j_{k-1}$. For $t=0$, we have $V_{k}$ itself, and it has $b_{0}$ apparent double points. For $t=1$, the projection $V_{k}^{\prime}$ in $S_{2 k}$ has $b_{0}$ improper double points and an apparent double curve of order $b_{1}$. From a general point of $S_{2 k}$ we can draw $j_{0}$ tangent lines to $V_{k}^{\prime}$. Now if $t=2$, the projection $V_{k}^{\prime \prime}$ in $S_{2 k-1}$ has a double curve of order $b_{1}$ on which lie $j_{0}$ pinch points and an apparent double surface of order $b_{2}$, and is such that the cone of the $\infty^{1}$ tangent lines drawn from a general point of $S_{2 k-1}$ is of order $j_{1}$. And so on for the other values of $t$. For $t=k$, the projection $V_{k}^{(k)}$ in $S_{k+1}$ has a double variety $V_{k-1}^{b_{k-1}}$ and a pinch variety $V_{k-2}^{j_{k-2}}$ lying on $V_{k-1}^{b_{k-1}}$. The characteristic $j_{k-1}$ is the order of the tangent $k$-dimensional cone and it is also the class of the curve in which a plane of $S_{k+1}$ meets $V_{k}^{(k)}$.

There are numerous other characteristics of the variety $V_{k}$ such as the orders of its various manifolds of multplicities higher than 2 and the ranks of its different sections by subspaces of $S_{2 k+1}$. With these we are not at present concerned and we are here concerned only with the $b$ 's and the $j$ 's just enumerated. Now we take the case $k=3$, that is, the $V_{3}$ in $S_{7}$.

By the method of complete degeneration we regard the four hypersurfaces of orders $n_{1}, n_{2}, n_{3}, n_{4}$ in $S_{7}$ which intersect in the $V_{3}$ we are studying as being composed entirely of hyperplanes: $A_{1}, A_{2}, \cdots, A_{n_{1}} ; B_{1}, B_{2}, \cdots, B_{n_{2}} ; C_{1}, \cdots, C_{n_{3}}$; $D_{1}, \cdots, D_{n_{4}}$. The $V_{3}$ of intersection is then composed of $n=n_{1} n_{2} n_{3} n_{4} \quad 3$-spaces $\left(A_{i_{1}} B_{i_{2}} C_{i_{3}} D_{i_{4}}\right)\left[i_{j}=1,2, \cdots, n_{j}\right]$. We write $\left(x_{1} x_{2} x_{3} x_{4}\right)$ in place of $\left(A_{i_{1}} B_{i_{2}} C_{i_{3}} D_{i_{4}}\right)$. This symbol for any set of particular values of the $x$ 's represents a particular $S_{3}$ belonging to the decomposed $V_{3}$. Thus, the set (1432) represents the $S_{3}$ common to $A_{1}, B_{4}, C_{3}, D_{2}$. The totality of all the $n=n_{1} n_{2} n_{3} n_{4}$ sets $\left(x_{1} x_{2} x_{3} x_{4}\right)$ for all the integral values of $x_{i}$ from 1 to $n_{i}[i=1,2,3,4]$ will be taken to be the symbolic representation of $V_{3}$.

To deal with the different apparent double varieties of $V_{3}$ is to deal with the different kinds of pairs of $S_{3}$ 's of the decomposed $V_{3}$, that is, with the different kinds of pairs of sets of values 
$\left(x_{1} x_{2} x_{3} x_{4}\right)$. There are in all $n(n-1) / 2$ pairs and they fall under four types. Under type I we have all those pairs each of which is such that the elements or $x$ 's of one set are all different from the corresponding elements or $x$ 's of the other, as for example (1111), (2324). Any such pair represents a pair of non-incident $S_{3}$ 's belonging to the decomposed $V_{3}$ and the number of such pairs is, as can easily be verified,

$$
2^{3}\left(\begin{array}{c}
n_{1} \\
2
\end{array}\right)\left(\begin{array}{c}
n_{2} \\
2
\end{array}\right)\left(\begin{array}{c}
n_{3} \\
2
\end{array}\right)\left(\begin{array}{c}
n_{4} \\
2
\end{array}\right) .
$$

Now type II consists of all those pairs in each of which three elements of one set are all different from the three corresponding elements of the other. Examples are (1111), (1223); (1234), (2414). A pair of this kind represents two $S_{3}$ 's in the decomposed $V_{3}$ having a point in common. The number of pairs belonging to this type is given by the symmetric function

$$
2^{2} \sum n_{1}\left(\begin{array}{c}
n_{2} \\
2
\end{array}\right)\left(\begin{array}{c}
n_{3} \\
2
\end{array}\right)\left(\begin{array}{l}
n_{4} \\
2
\end{array}\right) \text {. }
$$

For type III we have all those pairs each consisting of sets two of whose corresponding elements are different and two alike, as (1111), (1123). There are

$$
2 \sum n_{1} n_{2}\left(\begin{array}{l}
n_{3} \\
2
\end{array}\right)\left(\begin{array}{l}
n_{4} \\
2
\end{array}\right)
$$

such pairs each representing two $S_{3}$ 's in the decomposed $V_{3}$ with a line in common.

The remaining

$$
\sum n_{1} n_{2} n_{3}\left(\begin{array}{c}
n_{4} \\
2
\end{array}\right)
$$

pairs of sets, that is, all those pairs each having three elements in one set and the three corresponding elements in the other alike, are said to belong to type IV. A pair of this type represents a pair of $S_{3}$ 's having a plane in common and belonging to the decomposed $V_{3}$.

Now from a given point in $S$ - only one line can be drawn incident with two non-incident $S_{3}$ 's. We say that these two $S_{3}$ 's have a point of apparent intersection. The number of pairs of nonincident $S_{3}$ 's in the decomposed $V_{3}$ is the number of apparent double points of $V_{3}$ before decomposition. Being equal to the 
number of pairs of sets of values of the $x$ 's belonging to type I, it is given by

$$
b_{0}=2^{3}\left(\begin{array}{c}
n_{1} \\
2
\end{array}\right)\left(\begin{array}{c}
n_{2} \\
2
\end{array}\right)\left(\begin{array}{c}
n_{3} \\
2
\end{array}\right)\left(\begin{array}{c}
n_{4} \\
2
\end{array}\right) .
$$

Projecting the decomposed $V_{3}$ on to an $S_{5}$, we find that all those pairs of $S_{3}$ 's both non-incident and incident in a point project into pairs of $S_{3}$ 's each having an actual line of incidence. All the lines so obtained form the degenerate double curve in the projection of the decomposed $V_{3}$. Hence, the order of the double curve on the projection $V_{3}^{\prime \prime}$ of $V_{3}$ before degeneration is, from the results obtained for type I and type II,

$$
b_{1}=2^{3}\left(\begin{array}{c}
n_{1} \\
2
\end{array}\right)\left(\begin{array}{c}
n_{2} \\
2
\end{array}\right)\left(\begin{array}{c}
n_{3} \\
2
\end{array}\right)\left(\begin{array}{c}
n_{4} \\
2
\end{array}\right)+2^{2} \sum n_{1}\left(\begin{array}{c}
n_{1} \\
2
\end{array}\right)\left(\begin{array}{c}
n_{2} \\
2
\end{array}\right)\left(\begin{array}{c}
n_{3} \\
2
\end{array}\right) .
$$

A pinch point on $V_{3}^{\prime \prime}$ is given by a tangent plane of $V_{3}$ passing through a given line $l$ in $S_{7}$. If $V_{3}$ is completely degenerated, the given line $l$ and the point of incidence of the two $S_{3}$ 's of a pair represented by a pair of sets of type II determine a plane which is to be regarded as two tangent planes of $V_{3}$ passing through $l$. Therefore, we have

$$
j_{0}=2^{3} \sum n_{1}\left(\begin{array}{c}
n_{2} \\
2
\end{array}\right)\left(\begin{array}{c}
n_{3} \\
2
\end{array}\right)\left(\begin{array}{c}
n_{4} \\
2
\end{array}\right)
$$

for the number of pinch points on the projection $V_{3}^{\prime \prime}$ in $S_{5}$.

Now a given plane of $S_{7}$ and the line common to two $S_{3}$ 's of a pair whose representation belongs to type III determine $\infty^{1}$ 3-spaces each of which is to be considered as two tangent 3spaces of $V_{3}$. If $V_{3}$ is projected on to an $S_{4}$, every such tangent 3 -space meets $S_{4}$ in a pinch point of $V_{3}{ }^{\prime \prime \prime}$. The order of the locus of pinch points on $V_{3}^{\prime \prime \prime}$ is twice the number of pairs of sets of the $x$ 's belonging to type III and hence we have

$$
j_{1}=2^{2} \sum n_{1} n_{2}\left(\begin{array}{c}
n_{3} \\
2
\end{array}\right)\left(\begin{array}{c}
n_{4} \\
2
\end{array}\right) \text {. }
$$

In the projection in $S_{4}$ of the decomposed $V_{3}$ we find that the number of planes each of which is the projection of the plane of apparent intersection of the $S_{3}$ 's of a pair whose representation belongs to either type I or II or III is the order of the double surface on $V_{3}^{\prime \prime \prime}$ and is given by 


$$
\begin{gathered}
b_{2}=2^{3}\left(\begin{array}{c}
n_{1} \\
2
\end{array}\right)\left(\begin{array}{c}
n_{2} \\
2
\end{array}\right)\left(\begin{array}{c}
n_{3} \\
2
\end{array}\right)\left(\begin{array}{c}
n_{4} \\
2
\end{array}\right)+2^{2} \sum n_{1}\left(\begin{array}{c}
n_{2} \\
2
\end{array}\right)\left(\begin{array}{c}
n_{3} \\
2
\end{array}\right)\left(\begin{array}{c}
n_{4} \\
2
\end{array}\right) \\
+2 \sum n_{1} n_{2}\left(\begin{array}{c}
n_{3} \\
2
\end{array}\right)\left(\begin{array}{c}
n_{4} \\
2
\end{array}\right) .
\end{gathered}
$$

It is not difficult to see that the order $j_{2}$ of the tangent 3-dimensional cone of $V_{3}{ }^{\prime \prime \prime}$ is twice the number of pairs in type IV and we have

$$
j_{2}=2 \sum n_{1} n_{2} n_{3}\left(\begin{array}{c}
n_{4} \\
2
\end{array}\right)
$$

Incidentally we have the obvious relations

and

$$
2 b_{1}=2 b_{0}+j_{0}, \quad 2 b_{2}=2 b_{1}+j_{1}=2 b_{0}+j_{0}+j_{1}
$$

$$
n(n-1)=2 b_{2}+j_{2}=2 b_{0}+j_{1}+j_{2} .
$$

It is to be noticed that if one of the four $n$ 's is unity, then $b_{0}=0$, that is, if $V_{3}$ is the complete intersection of three general $V_{5}$ 's in an $S_{6}$, it cannot have improper double points. If two of the four $n$ 's are equal to unity, then we have, in addition to $b_{0}$ vanishing, $b_{1}=0$ and $j_{0}=0$. This means that a $V_{3}$ of complete intersection of two general $V_{4}$ 's in an $S_{5}$ has no double curve nor pinch points.

Now we write the results for the $b$ 's and the $j$ 's of $V_{k}$ in $S_{r}$ for $r \geqq 2 k+1$. If $V_{k}$ is the complete intersection of $r-k$ general hypersurfaces of orders $n_{1}, n_{2}, \cdots, n_{r-k}$ respectively, we find by arguments exactly similar to those for the case $k=3$ the following formulas:

$$
\begin{gathered}
b_{k-q}=\sum_{i=1}^{j-k-q} 2^{q-1+i} \sum n_{1} n_{2} \cdots n_{r-k-q-i}\left(\begin{array}{c}
n_{r-k-q-i+1} \\
2
\end{array}\right) \\
\cdot\left(\begin{array}{c}
n_{r-k-q-i+2} \\
2
\end{array}\right) \cdots\left(\begin{array}{c}
n_{r-k} \\
2
\end{array}\right),
\end{gathered}
$$

and

$$
j_{k-q}=2^{q} \sum n_{1} n_{2} \cdots n_{r-k-q}\left(\begin{array}{c}
n_{r-k-q+1} \\
2
\end{array}\right)\left(\begin{array}{c}
n_{r-k-q+2} \\
2
\end{array}\right) \cdots\left(\begin{array}{c}
n_{r-k} \\
2
\end{array}\right) .
$$

For $q=1$, we have 


$$
\begin{aligned}
b_{k-1} & =\sum_{i=1}^{r-k-1} 2^{i} \sum n_{1} n_{2} \cdots n_{r-k-1-i}\left(\begin{array}{c}
n_{r-k-i} \\
2
\end{array}\right)\left(\begin{array}{c}
n_{r-k-i+1} \\
2
\end{array}\right) \cdots\left(\begin{array}{c}
n_{r-k} \\
2
\end{array}\right) \\
& =\frac{1}{2} n_{1} n_{2} \cdots n_{r-k}\left(n_{1} n_{2} \cdots n_{r-k}-\sum n_{1}+r-k-1\right),
\end{aligned}
$$

which is the number of apparent double points on the curve in an $S_{3}$ into which the curve of intersection of $V_{k}$ by an $S_{r-k+1}$ is projected, and

$$
j_{k-1}=2 \sum n_{1} n_{2} \cdots n_{r-k-1}\left(\begin{array}{c}
n_{r-k} \\
2
\end{array}\right)=n_{n} \cdots n_{r-k} \sum\left(n_{1}-1\right),
$$

which is the rank of the curve of intersection of $V_{k}$ by an $S_{r-k+1}$.

By assigning to $q$ all the different values from 1 to $k$, we obtain all the formulas for the $2 k$ characteristics $b_{0}, b_{1}, \cdots, b_{k-1}$; $j_{0}, j_{1}, \cdots, j_{k-1}$. We have also the relations

and

$$
\begin{aligned}
& 2 b_{k-q}=j_{k-q-1}+2 b_{k-q-1} \\
& =j_{k-q-1}+j_{k-q-2}+2 b_{k-q-2} \\
& =j_{k-q-1}+j_{k-q-2}+\cdots+j_{1}+j_{0}+2 b_{0} \text {, }
\end{aligned}
$$

$$
n(n-1)=j_{k-1}+j_{k-2}+\cdots+j_{k-q}+2 b_{k-q} .
$$

We wish to add that, if $r<2 k+1$ and if $2 k+1-r=d$, we have $b_{0}=b_{1}=\cdots=b_{d-1}=0$ and also $j_{0}=j_{1}=\cdots=j_{d-2}=0$.

The University of California 